\title{
Control of a robotic prosthesis simulation by a closed-loop intracortical brain-machine interface
}

\author{
Dorian Goueytes, Aamir Abbasi, Henri Lassagne, Daniel E. Shulz, Luc Estebanez and Valérie Ego- \\ Stengel
}

\begin{abstract}
Closed-loop brain-machine interfaces may help restore the autonomy of amputees and tetraplegic patients. However, additional efforts are needed towards their realworld use with prostheses. Here we have interfaced a highly versatile closed-loop mouse BMI with an online model of a realworld prosthetic arm. We describe this setup and illustrate how it allows to explore the efficiency of different input and output coding strategies given a realistic modelling of the interactions between a commercial bidirectional prosthesis and its environment.
\end{abstract}

\section{INTRODUCTION}

Invasive Brain-Machine Interfaces (BMIs) are devices that access in real-time neuronal activity inside the brain in order to execute an action. Beyond research applications, this direct interfacing with the brain constitutes an opportunity to help patients suffering from severe motor damage, including paralysis and amputation cases. The architecture of invasive BMIs combines a neuronal recording device, usually multiple microelectrodes implanted in the brain, with an online analysis of the signal to extract commands that can be passed to an effector. This simple, one-directional strategy has already shown impressive results during the past years, successfully giving control of complex robotic prostheses to patients with heavy disabilities [1], [2].

However, emulating the fast, accurate control seen in natural movements remains a challenge. This is in part because explicit feedback mechanisms are often lacking. Indeed, on top of the eye contact with the prosthesis, the user also needs feedback about non-visual properties, including internal forces or occluded touch [3]. This is especially true for manipulation of complex, multi-joint prostheses. Moreover, somatosensory and proprioceptive feedback are of utmost importance to induce embodiment and sense of agency, which in turn impacts motor performance [4]. Recently, efforts have been made to incorporate somatosensory or proprioceptive-like feedback to BMIs, using approaches ranging from fully biomimetic stimulations of sensory cortices [5] to learning-based methods using arbitrary patterns of stimulation [6].

We have previously described a closed-loop BMI setup [7] based on the spiking activity of neurons recorded by chronically implanted electrodes in the primary motor cortex.

*Research supported by ANR Neurowhisk; by Labélisation FRM DEQ20170336761 and by the iCODE project funded by the IDEX ParisSaclay, ANR-11-IDEX-0003-02.

All authors are with Unité de Neurosciences, Information et Complexité, Centre National de la Recherche Scientifique, FRE 3693, 91198 Gif-surYvette, France (phone: +33(0) 1698234 46; e-mail: ego@unic.cnrs-gif.fr).
This system includes a sensory feedback using a high-speed projector to generate arbitrary photostimulation patterns over the somatosensory cortex of transgenic mice expressing channelrhodopsin (EMX-Cre;Ai27 strain, [8]). But so far this promising bidirectional BMI setup has not been tested in the context of interactions with a real-world prosthesis, although this could potentially challenge the usability of the system.

In particular, one issue that may prevent successful control of current brain-machine interfaces is the inherent delay between the readout of the motor command and the feedback. Motor BMIs, as complex systems, introduce new sources of latencies: an algorithmic delay, which duration depends on the complexity of the transformation between the recorded signal and the command output; and a second delay during the actual control of the prosthesis when it involves an additional driver layer, such as a proportional-integralderivative controller [9]. Finally, the online production of feedback by the BMI and its transduction into neuronal activity also takes time. These many sources of delays are likely to lead to a steep decrease in performance and learning, particularly in terms of error correction and accuracy [10].

Beyond latencies, the control of a real-world prosthesis raises many other challenges, in particular related to the specific geometry of the prosthesis actuators, which constraints the structure of the motor commands as well as of the feedback. Here we describe how we have attached a simulation of a commercial prosthesis to our BMI setup, and how this setup allows us to explore and test solutions to these challenges.

\section{METHODS}

\section{A. Surgical preparation}

All animal experiments were carried out using EMXCre;Ai27 mice that express channelrhodopsin 2 in their pyramidal neurons. In these mice, blue light illumination triggers spiking discharges in cortical neurons. We implanted the bidirectional BMI interface in two steps. First, to allow photostimulation of the cortical surface, we implanted a 5 $\mathrm{mm}$ diameter optical window over the primary somatosensory cortex (S1). During the same surgery, we also implanted a headpost to hold the mouse in place during the awake experiments. After two weeks of recovery, we used intrinsic imaging to confirm that the representation of whiskers in S1 (the so-called barrel cortex) was indeed located below the glass window. Finally, we implanted the mice with a 32-channel Neuronexus extracellular electrode in the whisker primary motor cortex (M1). After one week of recovery, the mouse was ready to be trained in the BMI task. 


\section{B. Overview of the system}

Our BMI system (Fig. 1a) is run by a main "master" software providing a graphical user interface and a hub that connects and streams data between three players: (1) the Neural Signal Processor (Cerebus NSP, BlackRock Microsystem), (2) the V-REP simulation software [11], and (3) the DLP projector (Vialux High-speed V7001). The NSP contains a hard real-time computer and performs online spike extraction and sorting from the $30 \mathrm{kHz}$ sampled continuous signal coming from the chronic electrode. This spike sorted data is streamed to the master hub. The DLP projector sends patterned light to a $2.5 \mathrm{~mm}^{2}$ area of the cortex as highdefinition frames $(1024 \times 768$ pixels $)$ with a $100 \mathrm{~Hz}$ refresh rate. Finally, the robotic software models an existing prosthesis, the Jaco2 arm from KINOVA, and simulates the interaction of this robotic arm with a virtual environment. The movements of the robotic arm are driven by the neuronal activity streamed from the NSP, and feedback from the robot controls the patterns of photostimulation sent back to the mouse.
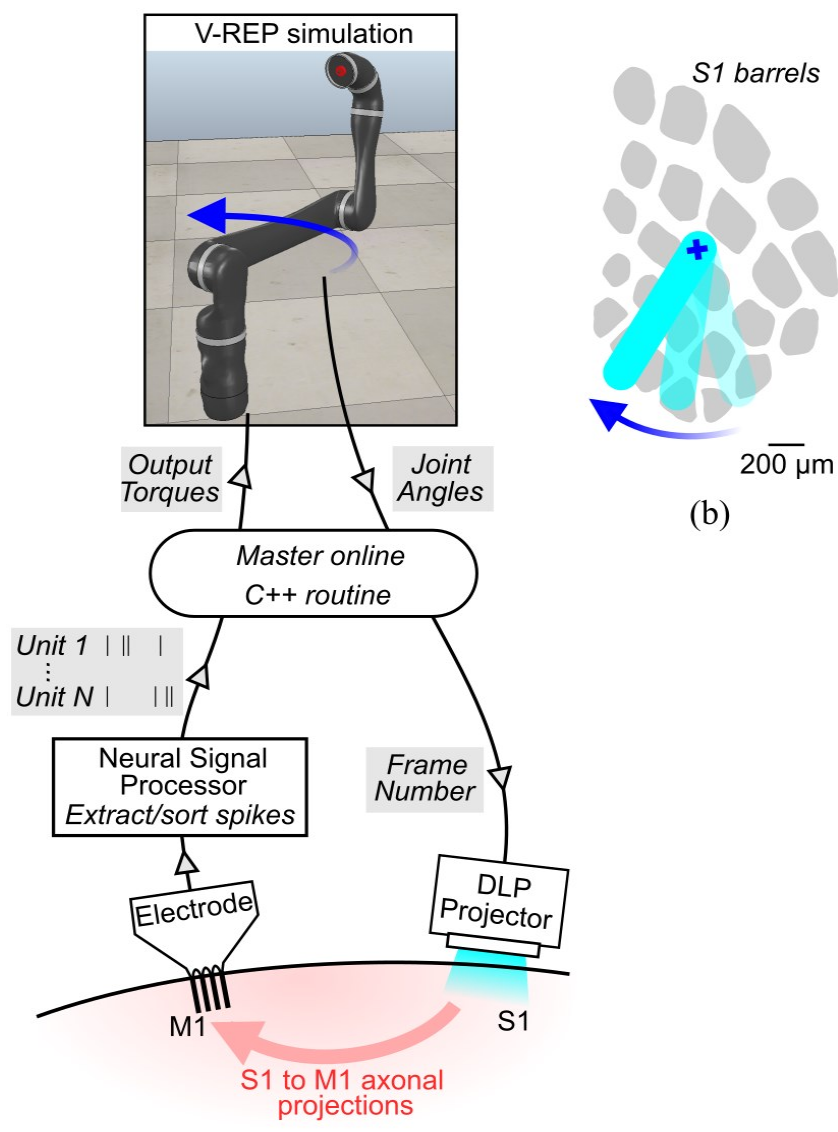

(b)

(a)

Fig. 1. (a) Information flow through the different components of the closed-loop BMI and prosthesis model. (b) Example of a feedback photostimulation on the mouse barrel cortex. An illumination bar rotates (arrow) around a fixed point (dark blue cross).

\section{Master Software}

In order to interface the different components of our system and ensure a proper flow of information during runtime, we developed a multi-threaded application in $\mathrm{C}++$ using the Qt environment. This choice was driven by the need to be compatible with the APIs of all other components of the system, as well as by the aim to maximize performance.

The main thread converts the firing rate into a motor command through a transfer function, monitors the state of the prosthesis and sends the appropriate pattern of photostimulation to the DLP projector. A second thread supports the graphical interface that provides a feedback on the status of the experiment as well as ways to adjust its parameters on the fly.

\section{Conversion of spiking rates into torques}

The prosthesis includes joints that should be actuated in both directions. Here we aimed to control one joint and ensure that the mouse can move it as easily in one direction as in the other. This type of symmetrical control is hard to derive from spiking activity. To address this challenge we implemented an antagonist control strategy, meaning that the joint was placed under the control of two groups of neurons that "pushed" in opposite direction. This strategy is inspired by a recent publication [12] showing that it ensures a nonbiased bidirectional control. We used a "greedy" selection algorithm to automatically select two groups of neurons of similar size and with similar firing rate. Those groups were fixed for the whole behavioural sequence across days. Their activity was dynamically weighted at the beginning of every control session depending on a 10 minutes baseline recording in order to avoid the possible build-up of a bias towards one direction due to day-to-day variability.

The actual command applied on the joint was generated from the spiking of these two groups by convoluting their activity with a $100 \mathrm{~ms}$ kernel, then translating these two instantaneous firing rate series into torque values, using a logistic function. The difference between these two opposite torques was finally applied to the elbow joint (Fig. 2). We chose to use a biomimetic torque-based control with the hope that learning would be facilitated in these more physiological conditions. Also, it removed the need to include a driver layer such as a PID, which would be necessary for a speed-based control.

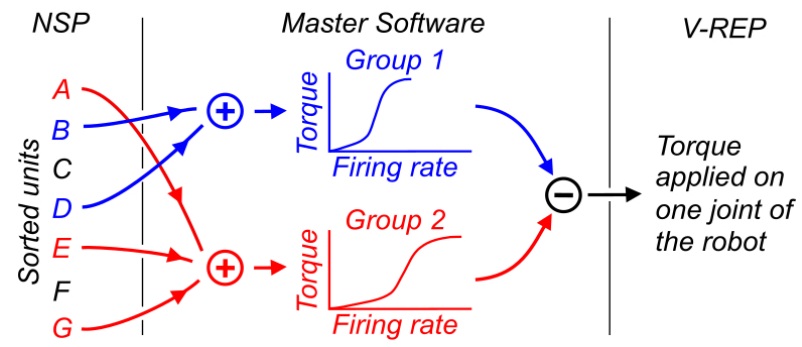

Fig. 2. The activity of two groups of neurons is merged, transformed into torques that are applied in opposite direction on the same joint. 


\section{E. Interfacing the $V$-REP robotic modelling software}

We used the V-REP native remote $\mathrm{C}++$ API to interface our master software with V-REP. It sends the torque commands to the robotic arm model, and reports to the master software the contacts of the arm with the virtual environment. The communication between the two programs works in a similar manner to a client-remote server system timed with a clock. Every time a torque command was sent from the master software, V-REP executed a $5 \mathrm{~ms}$ simulation step and returned the state of the prosthesis (posture, contact with the environment), which was then used by the master software to update the photostimulation feedback.

\section{F. Providing structured feedback to the mouse}

The DLP device displays blue light, arbitrary 2D photostimulation patterns at the surface of the cortex, which are updated every $10 \mathrm{~ms}$ with a power of $25 \mathrm{~mW} \cdot \mathrm{mm}^{-2}$. This configuration allows to explore a very broad range of feedbacks, depending on the type of prosthesis and on the decision to use or not a biomimetic approach.

Guided by the circular nature of the geometry of the prosthesis actuator, we chose to provide feedback to the mouse in the form of the angular position of the prosthesis joint. We encoded the state of the prosthesis as a rotating bar centered on the barrel cortex, and more precisely on the representation of the $\mathrm{C} 2$ whisker as determined by the optical intrinsic imaging. The bar thus followed the position of the joint (Fig. 1b).

\section{RESULTS}

Here we describe work to expand a mouse closed-loop BMI that we developed previously [7]. This brain-machine interface was composed of an invasive readout of the spiking activity in $\mathrm{M} 1$, and an artificial sensory feedback via the spatially and temporally structured photoactivation of Channelrhodopsin-expressing neurons in S1. We connected this interface to a robotic simulation engine, V-REP, which simulated a 7-degree of freedom arm, the Jaco2 from KINOVA robotics (Fig. 1). To validate the full setup, we focused on a single axis of the robot, corresponding to the first joint after its attachment base. We implemented the translation of spiking activity into torque signals that were relevant to control the robotic joint. On the sensory side, the angular position of the joint was fed back to the mouse as a structured pattern of cortical activation.

\section{A. Calibration of the closed-loop latency}

To evaluate the performance of our closed-loop system, we measured two complementary metrics. First we looked at the end-to-end hardware latency, which is the time between the occurrence of a spike recorded by the electrode and the

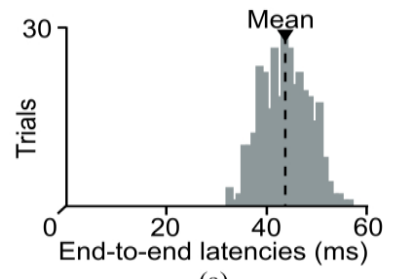

(a)

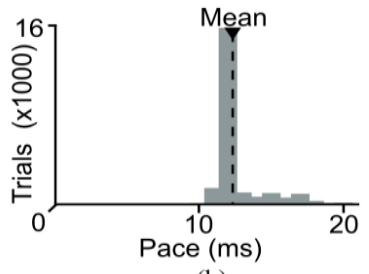

(b)
Fig. 3. (a) End-to-end latency of the BMI closed-loop. (b) Pace of the loop. resulting update of the photostimulation feedback. This measure represents the delay induced by all computations and transmissions within our BMI system, and is independent of the behaviour of the animal or the state of the cortex. Second we measured the pace at which the main thread of the master software operated, by measuring with a photodiode the timing of the transition between consecutive photostimulations.

The end to end latency of the main loop averaged $43.82 \mathrm{~ms}$ (SD $4.80 \mathrm{~ms}$, Fig. 3a), while the loop ticked at an average pace of $12.48 \mathrm{~ms}$ ( $\mathrm{SD}=2.07 \mathrm{~ms}$, Fig. 3b). These figures are larger than the values obtained in the absence of the simulation (average end-to-end latency $12.3 \mathrm{~ms}$, SD 1.03 , [7]) but they are still an improvement compared to the values obtained in some motor only protocols, as discussed in the introduction of [9].
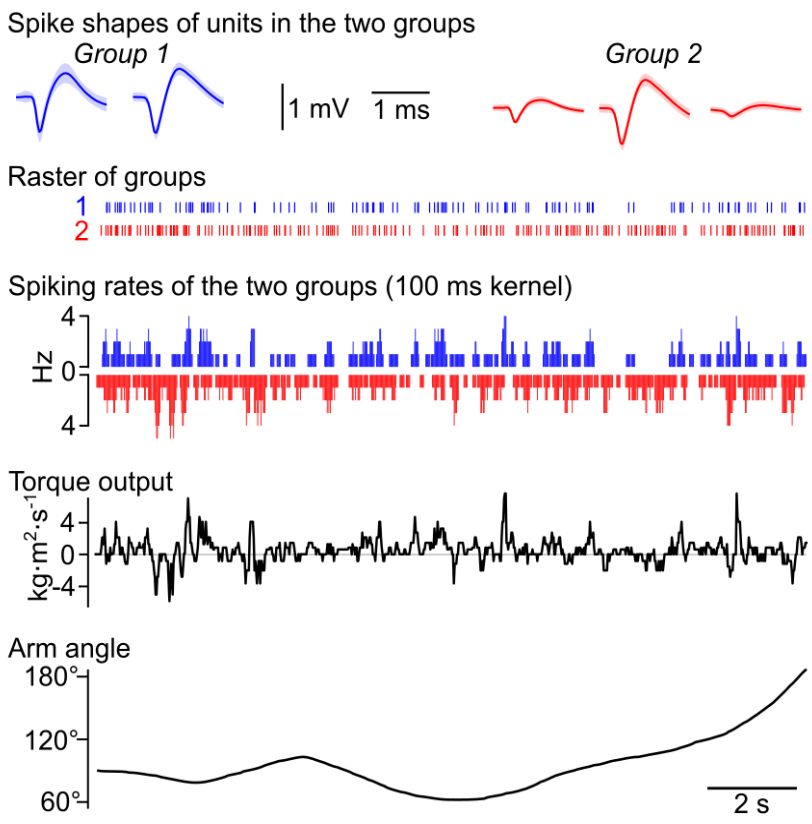

(a)

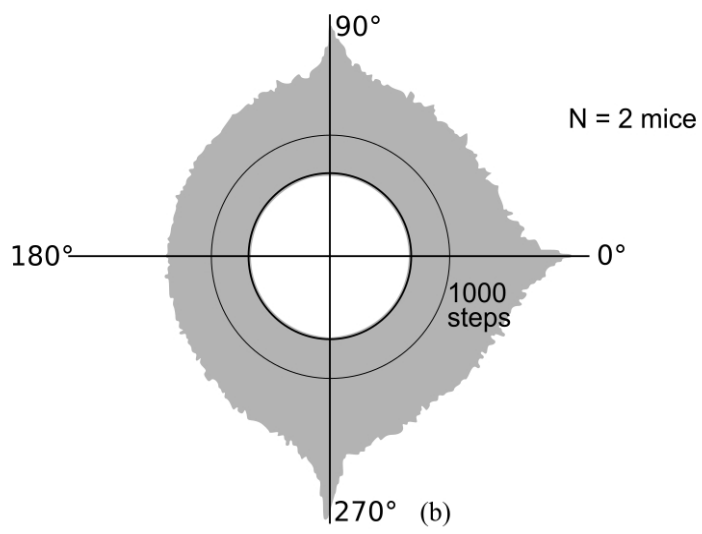

Fig. 4. (a) Example of the transformation of the raw spiking of two antagonist groups of neurons into movements of a robot joint. (b) Distribution of the position of the joint controlled by the BMI during the last five training sessions in two habituated mice. Starting angle of the joint is either $90^{\circ}, 0^{\circ}$ or $270^{\circ}$. 


\section{B. Control of the robotic arm}

Two mice were connected to our closed-loop BMI during 5 days, for 30 minutes two times per day (mean 57.5 trials/session, mean trial duration 31.33s). The mice were first habituated to the head fixation, with the BMI apparatus disconnected, for 1 session. Then, during BMI sessions, the activity of units in M1 controlled the rotation of the joint of the Jaco2 robot closest to the arm base. Fig. 4a displays how the spiking activity of five isolated neurons controlled one joint of the simulated $\mathrm{Jaco}^{2}$ prosthesis while receiving the real-time feedback. In this particular experiment, one group of 2 neurons generated the positive torque while another group of 3 neurons generated the negative torque. The firing rate of each group was smoothed by a $100 \mathrm{~ms}$ box kernel before being fed to the transfer function and generating torques. The torques were then subtracted and used as the final output torque applied to the joint (mean output torque $8.483 \mathrm{~kg} \cdot \mathrm{m}^{2} \cdot \mathrm{s}^{-2}$ across all sessions). Importantly, when we looked at the movement of the arm generated by the animal, we found that the activity generated by the neurons recorded in M1 was sufficient to entrain rotations of the joint, which covered its full $360^{\circ}$ span, in the two mice that were involved in the experiments (Fig. 4b).

Overall, those observations illustrate the feasibility and functionality of interfacing our fast BMI with a robotic modelling simulation, and they show the possibility to train surgically implanted mice to drive the prosthetic arm through the BMI.

\section{DISCUSSION}

We recently developed a fast bidirectional mouse BMI that incorporates a rich photoactivation-based feedback [7]. Here we report on a system that makes this BMI interact with an online simulation of a robotic arm. The mice controlled one joint of the arm using their primary motor cortex activity, and the BMI provided a dynamic feedback about the angular position of the arm by delivering optogenetic stimulation to the primary somatosensory cortex. The full system operated properly and at low latency that will hopefully greatly facilitate learning and performance in our future experiments.

To develop this design, we chose to use V-REP for simulating the robotic actuator because it provides both adaptability and a physically accurate environment. Disadvantages compared to the in-house development of a $\mathrm{C}++$ routine that would simulate the arm dynamics include programming constraints and a potential slowdown of the loop. However, V-REP offers a mature and versatile modelling environment that would be hard to fully replicate. The environment it emulates has driven technical decisions that will remain relevant when connecting the BMI loop to a physical prosthesis. This includes the choice of a joint control based on antagonist groups of neurons, and the choice of a rotating bar feedback. In addition, the networking constraints in V-REP are close to those encountered when working with real-world prosthesis, and addressing them early in our work will help us to seamlessly move from a virtual to a real actuator in the future.

This setup will be instrumental to systematically explore the extremely large space of input and output parameters that characterize invasive closed-loop BMIs. On the feedback side, our setup should allow to test different types of feedback structure beyond the rotating bar presented here, as well as different cortical locations. For example, we hope to determine whether biomimetic vs non biomimetic feedback may be optimal for information delivery about the current state of a robotic limb. Also, the suitability of primary sensory cortices vs. higher-order and associative cortical areas could be tested. The same type of approach could be applied to the neuronal spiking readout, by comparing a strategy based on decoding of the spiking activity with a strategy based on adaptive learning of the neuronal networks. In particular, we are interested in determining the impact, on motor control and learning, of additional latencies that would be artificially injected in the BMI loop. Moreover despite the fact that we explored so far only a one degree of freedom task, both our control strategy and feedback structure were designed to scale-up to a higher number of degrees of freedom. This will allow us in the future to explore complex, high-dimensional motion within our BMI framework.

\section{ACKNOWLEDGMENT}

The authors would like to thank Patrick Parra for his help with the mechanical work, Guillaume Hucher and Aurélie Daret for her help with the animals.

\section{REFERENCES}

[1]L. R. Hochberg et al., "Reach and grasp by people with tetraplegia using a neurally controlled robotic arm," Nature, vol. 485, no. 7398 , pp. 372-375, May 2012.

[2]A. B. Ajiboye et al., "Restoration of reaching and grasping movements through brain-controlled muscle stimulation in a person with tetraplegia: a proof-of-concept demonstration," The Lancet, Mar. 2017.

[3] R. L. Sainburg, M. F. Ghilardi, H. Poizner, and C. Ghez, "Control of limb dynamics in normal subjects and patients without proprioception," Journal of neurophysiology, vol. 73, no. 2, pp. 820-835, 1995.

[4]E. van den Bos and M. Jeannerod, "Sense of body and sense of action both contribute to self-recognition," Cognition, vol. 85, no. 2, pp. 177-187, Sep. 2002.

[5]S. J. Bensmaia, "Biological and bionic hands: natural neural coding and artificial perception," Philosophical Transactions of the Royal Society B: Biological Sciences, vol. 370, no. 1677, p. 20140209, Sep. 2015.

[6]M. C. Dadarlat, J. E. O’Doherty, and P. N. Sabes, “A learning-based approach to artificial sensory feedback leads to optimal integration," Nature Neuroscience, vol. 18, no. 1, pp. 138-144, Jan. 2015.

[7]A. Abbasi, D. Goueytes, D. E. Shulz, V. Ego-Stengel, and L. Estebanez, "A fast intracortical brain-machine interface with patterned optogenetic feedback," Journal of Neural Engineering, vol. 15, no. 4, p. 046011, Aug. 2018.

[8]L. Madisen et al., "A toolbox of Cre-dependent optogenetic transgenic mice for light-induced activation and silencing," Nature Neuroscience, vol. 15, no. 5, pp. 793-802, Mar. 2012.

[9]F. R. Willett, A. J. Suminski, A. H. Fagg, and N. G. Hatsopoulos, "Improving brain-machine interface performance by decoding intended future movements," Journal of Neural Engineering, vol. 10, no. 2, p. 026011 , Apr. 2013.

[10] S. Shimada, K. Hiraki, G. Matsuda, and I. Oda, "Decrease in prefrontal hemoglobin oxygenation during reaching tasks with delayed visual feedback: a near-infrared spectroscopy study," Cognitive Brain Research, vol. 20, no. 3, pp. 480-490, Aug. 2004.

[11] E. Rohmer, S. P. N. Singh, and M. Freese, "V-REP: A versatile and scalable robot simulation framework," in 2013 IEEE/RSJ International Conference on Intelligent Robots and Systems, Tokyo, 2013, pp. 13211326.

[12] R. M. Neely, A. C. Koralek, V. R. Athalye, R. M. Costa, and J. M. Carmena, "Volitional Modulation of Primary Visual Cortex Activity Requires the Basal Ganglia," Neuron, Mar. 2018. 